\title{
Rehabilitationsergebnisse von 766 Therapiebeendern in den Hamburger Sozialtherapeutischen Wohngemeinschaften für Drogenabhängige ${ }^{1}$
}

\section{G. Thiel \\ R. Ackermann \\ M. Prinzleve}

\section{Treatment Results of 766 Drug Users Undergoing Treatment in Therapeutic Communities in Hamburg}

\section{Zusammenfassung}

Studienziel: Es wurden die Therapieverläufe und -ergebnisse insbesondere die Integration in Arbeit/Ausbildung - in den Hamburger Sozialtherapeutischen Wohngemeinschaften für Drogenabhängige anhand des Selbstevaluationssystems der Einrichtungen ausgewertet. Weiterhin wurde überprüft, inwieweit Therapieergebnisse mit Klienten- oder Therapieprozessmerkmalen zusammenhingen. Untersuchungsgruppe: Alle Beender der stationären Abstinenztherapie der Jahre 1998-2002 im Projekt JORK-Jugendhilfe e.V. und bei Jugend hilft Jugend e.V. $(n=766)$. Die Untersuchungsgruppe war charakterisiert durch erhebliche psychosoziale Belastungen, z.B. ein Drittel ohne Hauptschulabschluss, drei Viertel ohne Berufsausbildung, im Mittel zwölfjährige Drogenabhängigkeit, zwei Drittel mit polyvalentem Drogenkonsum, fast drei Viertel mit Hafterfahrungen. Ergebnisse: Zwei Drittel aller Therapiebeender beendeten die Entwöhnungsphase regulär, vier von zehn die Gesamtrehabilitation (Entwöhnung und interne Adaption, Regelzeit: 10 Monate). Die mittlere Therapiezeit aller betrug 164 Tage, der regulären Therapiebeender 284 Tage. Bei Therapiebeendigung in der internen Adaption waren $73 \%$ im Mittel seit fast vier Monaten in Arbeit oder Ausbildung integriert, im letzten halben Jahr vor der Therapie waren es $25 \%$ (Effektgröße d = 1,11). Gemäß Klientenselbsteinschätzung verbesserten etwa die Hälfte bis drei Viertel ihre gesundheitliche Verfassung, ihre schulisch-berufliche und soziale Situation sowie die Wohnsituation deutlich bzw. sehr deutlich, drei Viertel stabilisierten sich in ihrer Persönlichkeit stark bzw. sehr stark. Etwa nach fünf bis sieben Therapiemonaten verdoppelte sich in verschiede-

\footnotetext{
1 Die Untersuchung wurde gefördert von der LVA Hamburg.
}

\section{Abstract}

Objective: The study evaluates treatment courses and treatment results - especially the integration into employment/education in the therapeutic communities in Hamburg using the self-evaluation system of the institutions. Moreover, potenzial relationships between treatment results and characteristics pertaining to clients or the process of treatment are investigated. Sample: All drug users who finished the residential drug treatment offered by 'Project Jork - Jugendhilfe e.V.' and 'Jugend hilft Jugend e.V.' between 1998 and $2002(n=766)$. The sample was characterised by a high degree of psychosocial problems. For example, one third had no formal education, three fourths had no professional education, mean length of drug dependence was twelve years, two thirds showed multiple drug use, and nearly three fourths had served a prison sentence. Results: Two thirds of all who finished the treatment regularly completed the rehabilitation, four out of ten the whole programme (rehabilitation plus adaptation phase, regular duration: ten months). Mean treatment duration was 164 days for the whole sample, and 284 days for those who regularly concluded the entire programme. Of those who finished treatment as planned, an average of $73 \%$ were in regular employment or education since four months at the end of the treatment, compared to $25 \%$ in the last six months prior to treatment entry (effect size $d=1.11$ ). According to clients' self-ratings, about half up to three fourths of them significantly improved their health status, their employment and social situation, and their living conditions, three fourths experienced a significant or very significant personality stabilisation. After five to seven months in treatment, there was a two-fold increase of improvements in several success criteria. Regular treatment concluders had better overall results than those who dropped out.

Institutsangaben

Projektgruppe Evaluation und Drogenforschung - Jugendhilfe e.V.

Projektteam Qualitätssicherung und Evaluation - Jugend hilft Jugend e.V.

Korrespondenzadresse

Dr. phil. Günter Thiel · Repsoldstr. 4 · 20097 Hamburg · E-mail: dr.guenter.thiel@t-online.de

Bibliografie

Suchttherapie 2004; 5: 180-190 ๑ Georg Thieme Verlag KG Stuttgart · New York

DOI 10.1055/s-2004-813623

ISSN $1439-9903$ 
nen Ergebniskriterien der Therapieerfolg. Die regulären Therapiebeender erzielten gegenüber den vorzeitigen durchweg bessere Therapieergebnisse. Die Art der Therapiebeendigung, die Integration in Arbeit/Ausbildung sowie weitere Ergebniskriterien korrelierten nicht oder nur sehr schwach mit Klientenmerkmalen. Die Therapiezeit sowie die Integration in Arbeit/Ausbildung in der Therapiemitte waren die stärksten Prädiktoren für verschiedene Therapieergebnisse. Klienten, mit denen häufiger mit Rückfall gearbeitet wurde, hatten keine schlechteren Therapieergebnisse erzielt. Schlussfolgerungen: Langjährig Drogenabhängige mit erheblichen psychosozialen Belastungen können in normale Arbeits- und Ausbildungsverhältnisse integriert werden. Eine Therapiezeit von 10 Monaten mit interner Adaption erscheint angemessen, um deutliche Fortschritte zu ermöglichen. Therapieergebnisse sind durch Klientenmerkmale nicht in relevantem Maße vorhersagbar. Stattdessen sollten Prozessvariablen als Prädiktoren für Therapieergebnisse stärker überprüft werden.

\section{Schliusselwörter}

Stationäre Drogentherapie · medizinische Rehabilitation · therapeutische Gemeinschaft - Integration in Arbeit · Abstinenztherapie
There was no or only a slight correlation found between clients' characteristics and the conclusion of treatment, integration into employment/education and other success criteria. Time in treatment and integration into employment/education while in treatment were the strongest predictors for several treatment results. Drug users who relapsed during treatment did not show worse results. Conclusions: It is possible to integrate long-term drug users with a high degree of psychosocial problems into regular employment or education. Treatment duration of ten months (including an internal adaptation) seems adequate to achieve significant improvements. Since clients' characteristics are of limited value to predict treatment results, further studies should focus on variables concerning the process of treatment.

\section{Key words}

Residential drug treatment · rehabilitation · therapeutic community · integration into employment · abstinence treatment

\section{Einleitung}

In den stationären deutschen Drogentherapieeinrichtungen beendete bis 1997 etwa gut ein Drittel mit einer Variation zwischen 19 und 49\% die stationäre Therapie „regulär“ oder „planmäßig“ [1 - 10]. In der umfangreichsten deutschen Studie von Küfner et al. [11] wurde ein Anteil regulärer Therapiebeendigungen von $28 \%$ in 41 Therapieeinrichtungen angegeben. Bei dieser globalen Aussage werden Unterschiede und Veränderungen in der Zusammensetzung des aufgenommenen Personenkreises, der Definition „regulärer Therapiebeendigung“, der finanzierten Regeltherapiezeit, des Anforderungsprofils der Therapieeinrichtungen, der Entlassungskriterien sowie der zum Untersuchungszeitraum gegebenen gesellschaftlichen Rahmenbedingungen nicht näher berücksichtigt.

Die Integration in Arbeit oder Ausbildung ist für viele suchtmittelabhängige Personen ein sehr wichtiger Schritt bei der Entwicklung einer anderen Lebensweise und im längerfristigen Prozess der Überwindung der Suchtmittelabhängigkeit [2, 12, 13]. Umso verwunderlicher ist es, dass in der fachlichen Diskussion erst in den letzten Jahren - u.a. auf dem 8. Heidelberger Kongress des Fachverbands Sucht (FVS) e.V. [14] - hervorgehoben wurde, dass sich Rehabilitationseinrichtungen verstärkt um die berufliche Eingliederung kümmern sollen. Im Regelfall war während der stationären Rehabilitation in den meisten Fachkliniken für Drogenabhängige zwar die Wiederherstellung der Erwerbsfähigkeit, jedoch nicht die reale Integration in Arbeit oder Ausbildung konzeptionell vorgesehen und so gab es in dieser Hinsicht nur sehr begrenzte Erfolge (z. B. [10]).

Mittlerweile fordern Rentenversicherungsträger, dass Rehabilitationseinrichtungen die berufliche Rehabilitation bereits während der Entwöhnungsbehandlung in Angriff nehmen und dass es von der „Erwerbsfähigkeit zur Erwerbstätigkeit“ kommen möge [15]. Dieser Grundansatz wird in den Hamburger Sozialtherapeutischen Wohngemeinschaften für Drogenabhängige seit 1970 praktiziert. In früheren Veröffentlichungen waren etwa 90\% der regulären Therapiebeender am Therapieende seit einigen Monaten in „normale“ Arbeits- oder Ausbildungsverhältnisse integriert $[6,7,9,17]$. Fast drei Viertel der regulären und etwa die Hälfte der „Spätabbrecher“ verbesserten ihre individuelle Integration in Arbeit oder Ausbildung deutlich gegenüber der Zeit vor der Therapie.

Vollmer und Ellgring [4], Roch u.a. [18] oder Küfner u.a. [11] stellten fest, dass Anamnese- oder Persönlichkeitsvariablen von Klienten in keinem, in keinem stabilen oder allenfalls schwachem Zusammenhang mit dem Therapieergebnis standen. In die gleiche Richtung gingen Ergebnisse in den Hamburger Sozialtherapeutischen Wohngemeinschaften $[9,17]$.

An schwachen bivariaten Einzelzusammenhängen zeigte sich in diesen Studien unter anderem, dass Klienten mit höherem Alter zu Therapiebeginn, höherem Einstiegsalter in den Drogenkonsum, abgeschlossener Berufsausbildung, vorhandener Arbeitserfahrung, besserer Schulbildung, ohne Heimaufenthalte, ohne Haftaufenthalte, ohne feste Partnerbeziehung zu Therapiebeginn, ohne Substitutionserfahrung, mit längeren Cleanzeiten, mit weniger starker Suchtmittelabhängigkeit, geringerer gesundheitlicher bzw. psychosozialer Belastung und Therapiebeginn mit justiziellem Druck häufiger die Therapie regulär beendeten.

In verschiedenen Studien war ein wesentliches Ergebnis, dass die reguläre Beendigung der stationären Therapie sowie die in der Therapie verbrachte Zeit das beste Kriterium zur Vorhersage des Therapieerfolgs seien [5, 18]. Die Bedeutung einer längeren The- 
rapiezeit als Prädiktor für erfolgreichere Rehabilitation wird auch in diversen angloamerikanischen Veröffentlichungen unterstrichen [19-22].

Einzelne Arbeiten untersuchten einzelne Aspekte des Therapieprozesses in ihrer Bedeutung für das Therapieergebnis: So hatten reguläre Therapiebeender seltener gegen das Abstinenzgebot während der Therapie verstoßen als die vorzeitigen [10, 11]. In den Hamburger Sozialtherapeutischen Wohngemeinschaften waren die regulären Therapiebeender bereits etwa in der Therapiemitte deutlich besser in Arbeit oder Ausbildung integriert [9].

In der vorliegenden Studie werden Evaluationsergebnisse der Hamburger Sozialtherapeutischen Wohngemeinschaften zu allen Therapieverläufen der Jahre 1998-2002 dargestellt. Die zentralen Fragestellungen waren dabei:

1. Welcher Personenkreis wird in die Hamburger Sozialtherapeutischen Wohngemeinschaften aufgenommen?

2. Welche Therapieergebnisse werden erreicht? Vor allem: In welchem Umfang gelingt die Integration in „normale“ $\mathrm{Ar}$ beits- oder Ausbildungsverhältnisse?

3. Gibt es Klienten- oder Therapieprozessmerkmale, die eine Prädiktion von Therapieergebnissen ermöglichen?

\section{Untersuchungsbeschreibung}

\section{Konzeptionelle Aspekte der beiden Reha-Einrichtungen}

$\mathrm{Zu}$ den besonderen Profilmerkmalen der Hamburger Sozialtherapeutischen Wohngemeinschaften für Drogenabhängige (Projekt JORK-Jugendhilfe e.V.; Jugend hilft Jugend e.V.) gehörten neben der Behandlung der Suchtproblematik in kleineren Therapeutischen Gemeinschaften die frühzeitige Einbeziehung gesellschaftlicher Realität und die Orientierung auf die Integration in „normale“ Arbeits- oder Ausbildungsverhältnisse als wesentlicher Schwerpunkt der Rehabilitation. Die Gesamttherapie (Regeldauer: 10 Monate) war strukturell untergliedert in eine erste Therapiestufe (Entwöhnungsphase) von etwa fünf Monaten und eine zweite Therapiestufe mit interner Adaptionsphase von etwa fünf Monaten Dauer. Anschließend erfolgte die ambulante Nachbetreuung. Der Aufenthalt in den jeweiligen Abschnitten der Rehabilitation richtete sich immer nach den individuellen Eingangsvoraussetzungen und dem Therapieverlauf, so dass kürzere oder längere Therapiezeiten konzeptionell möglich waren. In der ersten Therapiestufe fand ein tagesstrukturierendes Wochenprogramm mit personen- und themenzentrierten Gruppengesprächen, Einzelgesprächen und -beratung, gesundheitsfördernden, allgemeinbildenden und interessenweckenden Arbeitsgemeinschaften, Hausdiensten, Freizeitaktivitäten sowie mit individuell vereinbarten Aufgaben statt.

Die beruflich-schulische Orientierung beinhaltete die Auseinandersetzung mit den bisherigen Erfahrungen, die Erkundung realer Möglichkeiten, die Entscheidung für ein Ziel und entsprechende Bewerbungsaktivitäten. Dabei erfolgte in der ersten Therapiestufe die Unterstützung in einer Berufsorientierungsgruppe, über Einzelberatung und aktive Einzelfallhilfe. Bedingung für den Wechsel in die zweite Therapiestufe war - neben dem Erreichen anderer Therapieziele - ein Schul-, Ausbildungs-, Praktikums- oder Arbeitsplatz, der zumindest in absehbarer Zeit aufgenommen werden kann.

In der zweiten Therapiestufe stand der Einstieg in Arbeit/Ausbildung als Arbeits- und Belastungserprobung im Mittelpunkt. Die Bewältigung dieses für die meisten Klienten großen Schrittes wurde sozialtherapeutisch begleitet. Zusätzlich nahmen die Klienten nachmittags, abends und am Wochenende am therapeutischen Programm (Gruppengespräche, Einzelgespräche, Einzelberatung, Freizeitaktivitäten, Hausdienste) teil [23, 24].

Im Untersuchungszeitraum unterhielten die beiden Einrichtungen 80 Therapieplätze in insgesamt neun Wohngemeinschaften am Rande Hamburgs mit Anbindung an das öffentliche Verkehrsnetz. Die gruppentherapeutische Betreuung (Betreuungsschlüssel etwa 1:3) erfolgte durch multiprofessionelle Teams gleichberechtigter Mitarbeiter, vorwiegend Sozialpädagogen oder Mitarbeiter mit vergleichbarer Qualifikation und Ausbildung.

\section{Datenerhebung}

Bei der vorliegenden Untersuchung handelt es sich um eine naturalistische prospektive Studie. Es wurden alle Therapiebeender der Jahre 1998-2002 ( $\mathrm{n}=766$ ) der beiden Reha-Einrichtungen (JORK: $n=448$; Jugend hilft Jugend: $n=318$ ) einbezogen. Zwischen den einzelnen Jahren variierte die Zahl der Therapiebeender zwischen $n=136$ und $n=170$. Die Aufnahme in die Einrichtungen und die Durchführung der stationären Rehabilitation erfolgten nicht „standardisiert“, sondern so, wie es konzeptionell vorgesehen war mit entsprechenden Anpassungen im Untersuchungszeitraum. Alle wesentlichen Fragestellungen, die Erhebungsinstrumente und der Zeitpunkt der Datenerhebung standen zu Beginn der Studie fest. Von der maximalen Stichprobengröße gab es in einzelnen Variablen wegen missing data unerhebliche Abweichungen. Wenn einzelne Ergebnisse sich auf Teilstichproben beziehen, wird dieses dargestellt.

Zur Überprüfung der zentralen Fragestellungen wurden folgende Erhebungsinstrumente eingesetzt:

Aufnahmedokumentation: Erhebung von 65 Einzelmerkmalen in einem Aufnahmedokumentationsbogen (u.a. soziodemografische Merkmale, Therapieerfahrungen, justizielle Belastungen, Daten zum Suchtverlauf etc.) zu Therapiebeginn durch die Mitarbeiter der Einrichtungen im Rahmen der Aufnahmeanamnese.

Mitarbeiter-Therapieabschlussbogen: Bei Therapiebeendigung dokumentierte der für den jeweiligen Klienten zuständige Mitarbeiter die Therapieverlaufsergebnisse in einem Therapieabschlussbogen. Dieser enthielt zum einen „harte“ Daten (z.B. die Ergebnisse der Integration in Arbeit oder Ausbildung), zum anderen schätzten die Mitarbeiter die während der Therapie eingetretenen Veränderungen in den Merkmalen „Gesundheit“, „Arbeit/Ausbildung“, „Soziale Situation“, „Freizeitverhalten“, „Wohnsituation“ und „Persönlichkeitsstabilisierung“ anhand fünfstufiger Ratingskalen ein. 
Klienten-Feedbackbogen: Die Klienten schätzten in einem Feedbackbogen am Ende der Therapie die bei sich wahrgenommenen Veränderungen in den gleichen sechs Merkmalen wie die Mitarbeiter ebenfalls anhand der gleichen fünfstufigen Ratingskalen ein. Sie gaben zusätzlich an, wie wichtig die jeweiligen Veränderungen für sie waren.

Im Klienten-Feedbackbogen gaben die Klienten zusätzlich ihre Zufriedenheit mit den Therapieergebnissen an. Ab 2000 wurde der Klienten-Feedbackbogen um acht Items zur Beurteilung von Therapieprozessmerkmalen [58] ergänzt (z. B. „Ich hatte Gelegenheit, die für mich wichtigen Problembereiche zu bearbeiten.“ „Von den MitarbeiterInnen fühlte ich mich fachlich sehr gut betreut und beraten.“ „Ich fühlte mich von den MitarbeiterInnen als Person respektiert und geachtet.").

Die Beantwortung des Klienten-Feedbackbogens erfolgte freiwillig. Der Bogen wurde allen bei Beendigung der Therapie mit der Bitte um Beantwortung und Rücksendung bzw. Rückgabe an die Geschäftsstelle der Reha-Einrichtungen gegeben.

74\% hatten den Klienten-Feedbackbogen ausgefüllt, und zwar $83 \%$ der regulären und $45 \%$ der vorzeitigen Therapiebeender („Abbrecher“ und Entlassene) in der zweiten Therapiestufe. Die nähere Analyse ergab, dass die „responder" eine ausgelesene Teilgruppe mit etwas besseren Therapieergebnissen darstellten: Sie hatten eine längere Therapiezeit gehabt $(n=358)$, gemäß Mitarbeiter-Ratings im Mitarbeiter-Therapieabschlussbogen hatten sie größere Verbesserungen in verschiedenen Bereichen erzielt, bei Therapiebeendigung waren sie häufiger in Arbeit oder Ausbildung integriert und zogen häufiger in eigenen Wohnraum (jeweils mindestens $\mathrm{p}<.01$; Cramer-V=.15 -.30).

Datenauswertung und Hypothesen: Die vorliegenden Daten wurden von einer wissenschaftlichen Hilfskraft in eine Datenbank übertragen und einer systematischen Überprüfung und Fehlerbereinigung unterzogen, Umkodierungen sowie die Neuberechnung von Variablen wurden vorgenommen.

Bei der statistischen Auswertung der Daten wurden in der Regel bei Nominal- oder Ordinalskalenqualität nichtparametrische Verfahren angewandt, bei höherer Skalenqualität - oder wenn es bei Verletzung der entsprechenden Voraussetzungen eher zu „konservativen“ Ergebnissen kam - auch parametrische Tests. Bei einzelnen Ergebnissen wurden die Effektgrößen angegeben [25]. Die Auswertung erfolgte mit dem Programm SPSS 10.0. Es wurde bei zweiseitiger Fragestellung als statistisches Entscheidungskriterium $\mathrm{p}<.05$ gewählt.

Mit einseitiger Fragestellung wurden folgende Einzelhypothesen, die aus dem einleitend dargestellten Forschungsstand abgeleitet wurden, überprüft:

1. Es wurde generell angenommen, dass reguläre Therapiebeender - verglichen mit den vorzeitigen - bessere Ergebnisse erzielen.

2. Hinsichtlich des Therapieprozesses wurde angenommen, dass Klienten mit besserer Integration in Ausbildung/Arbeit am Anfang der zweiten Therapiestufe häufiger die Therapie regulär beenden und bessere Therapieergebnisse erzielen.
3. Eine längere Therapiezeit und weniger Rückfälle während der Therapie gehen einher mit besseren Therapieergebnissen.

Datenreduktion: Die sechs Ratingskalen des Mitarbeiter-Therapieabschlussbogens ließen sich faktorenanalytisch zu einer Generalfaktorlösung mit $62 \%$ Varianzaufklärung (erster Eigenwert 3,76; zweiter Eigenwert 0,67) zurückführen. Die Ladungen der Einzelskalen variierten zwischen .68 und .88 . Die berechneten Faktorwerte wurden als „Allgemeine Therapieverbesserungen aus Mitarbeitersicht“ interpretiert.

Die faktorenanalytische Datenreduktion der sechs Ratingskalen im Klienten-Feedbackbogen ergab ebenfalls eine Generalfaktorlösung mit 49\% Varianzaufklärung (erster Eigenwert 2,95; zweiter Eigenwert 0,80). Die Ladungen der Einzelskalen lagen zwischen .64 und .79. Der Faktor wurde als „Allgemeine Therapieverbesserungen aus Klientensicht" gedeutet.

Die Therapieprozessmerkmalskalen ließen sich zu einem Generalfaktor mit $49 \%$ Varianzaufklärung zusammenfassen (erster Eigenwert 3,93; zweiter Eigenwert 0,84). Die Ladungen der Einzelskalen variierten zwischen .55 und .80 . Die Faktorwerte wurden als „Therapieprozessmerkmale aus Klientensicht“ interpretiert [26].

\section{Ergebnisse}

\section{Deskription der Untersuchungsgruppe}

Die Therapiebeender waren in der Aufnahmedokumentation folgendermaßen näher zu beschreiben (gültige Fälle zwischen $\mathrm{n}=737$ und $\mathrm{n}=766$ ):

Der Frauenanteil lag bei $18 \%$. Die Männer waren im Mittel 29,6 Jahre $( \pm 6,2)$, die Frauen 28,1 Jahre $( \pm 6,8)$ alt. $30 \%$ waren 35 Jahre und älter, $7 \%$ jünger als 20 Jahre. $85 \%$ kamen aus Hamburg, ebenfalls $85 \%$ besaßen die deutsche Staatsangehörigkeit.

Die mittlere Dauer der Drogenabhängigkeit betrug 11,9 Jahre ( \pm 6.3$)$. Im Mittel waren die Klienten 39\% ( $\pm 16 \%$ ) ihrer Lebenszeit drogenabhängig. In einer Clusteranalyse unter Einbeziehung von Alkohol, Heroin, Kokain, Methadon, Benzodiazepinen und Cannabis konnten für $64 \%$ polyvalente Konsummuster im letzten halben Jahr vor der Therapie beschrieben werden, u.a. konsumierten 68\% Heroin, 65\% Kokain und 38\% Benzodiazepine. $17 \%$ hatten sich im letzten halben Jahr mindestens eine Überdosis verabreicht.

33\% hatten keinen Hauptschulabschluss, 45\% Hauptschulabschluss. $73 \%$ wiesen keine abgeschlossene Berufsausbildung auf, und $53 \%$ hatten allenfalls geringfügige Arbeitserfahrung. Bei denjenigen mit Arbeitserfahrung lag die letzte Arbeitstätigkeit im Mittel 28,3 Monate $( \pm 32.3)$ zurück.

$85 \%$ waren vorbestraft, $71 \%$ hatten Hafterfahrungen, im Mittel 30,3 Monate $( \pm 34,9)$. $65 \%$ begannen die Therapie mit einer Form juristischen Drucks (z. B. Bewährung; Weisung; §35 BtmG; ausstehendes Verfahren). 13 \% waren in ihrem Leben wegen einer 
psychotischen Problematik in einer psychiatrischen Einrichtung gewesen. Im Mittel hatten die Aufgenommenen 4,4 Krankenhausentzüge $( \pm 4,3)$ absolviert. Für $39 \%$ war es der erste stationäre Therapieversuch. Bei denjenigen mit stationärer Therapievorerfahrung betrug diese im Mittel 9,9 Monate $( \pm 9,5)$. 66\% waren in der Vergangenheit substituiert gewesen, davon wiederum $46 \%$ mit psychosozialer Betreuung.

75\% gaben zu Therapiebeginn gesundheitliche Probleme an, 66\% Hepatitis C. 29\% lebten in der Kindheit/Jugendzeit zeitweise im Heim. 36\% hatten mindestens einen Suizidversuch im Leben unternommen, $20 \%$ mehr als einen. $63 \%$ gaben schwere körperliche Gewalterfahrungen an, 50\% der Frauen sexuelle Gewalterfahrungen.

Im letzten halben Jahr vor der Therapie lebten 39\% in eigener Wohnung, $15 \%$ bei Angehörigen. $46 \%$ waren wohnungslos.

$9 \%$ waren verheiratet, $8 \%$ geschieden und $83 \%$ ledig. $34 \%$ hatten zumindest ein Kind. Vor der Therapie lebten $46 \%$ alleinstehend und $40 \%$ in festen Beziehungen. $43 \%$ hatten im letzten halben Jahr vor der Therapie keine oder nur seltene Kontakte zu drogenfreien Personen.

Im letzten halben Jahr vor der Therapie waren drei Viertel weder im Arbeits- noch im Ausbildungsbereich integriert gewesen.

57\% bezogen vor der Therapie Sozialhilfe, 18\% hatten Einkommen aus Erwerbstätigkeit. 86\% waren verschuldet, 25\% mit Schulden von $10000,-€$ und mehr.

Im Untersuchungszeitraum 1998-2002 hat es hinsichtlich der psychosozialen Situation bei Aufnahme der Therapie keine signifikanten Veränderungen gegeben. Ausnahmen: In den letzten beiden Jahren hatte sich der Anteil älterer Klienten (35 Jahre/älter) von 25\% (1998/1999) auf 35\% erhöht ( $\mathrm{p}<.01)$. Der tägliche Heroinkonsum vor der Therapie ging von 64\% (1998) auf $44 \%$ (2002) kontinuierlich zurück ( $\mathrm{p}<.05)$.

Die Therapiebeender der beiden Reha-Einrichtungen unterschieden sich zu Therapiebeginn nicht in u.a. folgenden Merkmalen: Alter, Geschlecht, Dauer der Drogenabhängigkeit, Konsumverhalten vor der Therapie, Partnersituation, Schul- und Berufsbildung, Arbeitserfahrung und berufliche Integration, Behandlungserfahrung, Psychiatrieaufenthalte, Heimaufenthalte, gesundheitliche Probleme. Die Klienten einer Einrichtung kamen etwas häufiger aus Hamburg, hatten etwas häufiger Hafterfahrungen und begannen die Therapie häufiger auf der Basis des $\S 35$ BtmG (jeweils $\mathrm{p}<.001)$.

\section{Therapieergebnisse}

\section{Therapieverläufe}

Unter Berücksichtigung aller Aufnahmen ab dem ersten Therapietag $(n=766)$ wechselten $64 \%(n=490)$ aus der ersten Therapiestufe (Entwöhnungsphase) in die interne Adaptionsphase, $38 \%(n=292)$ beendeten die Gesamttherapie einschließlich interner Adaption regulär (Abb.1). „Regulär“ bedeutete, dass die Klienten am Ende der bewilligten Therapiezeit planmäßig und

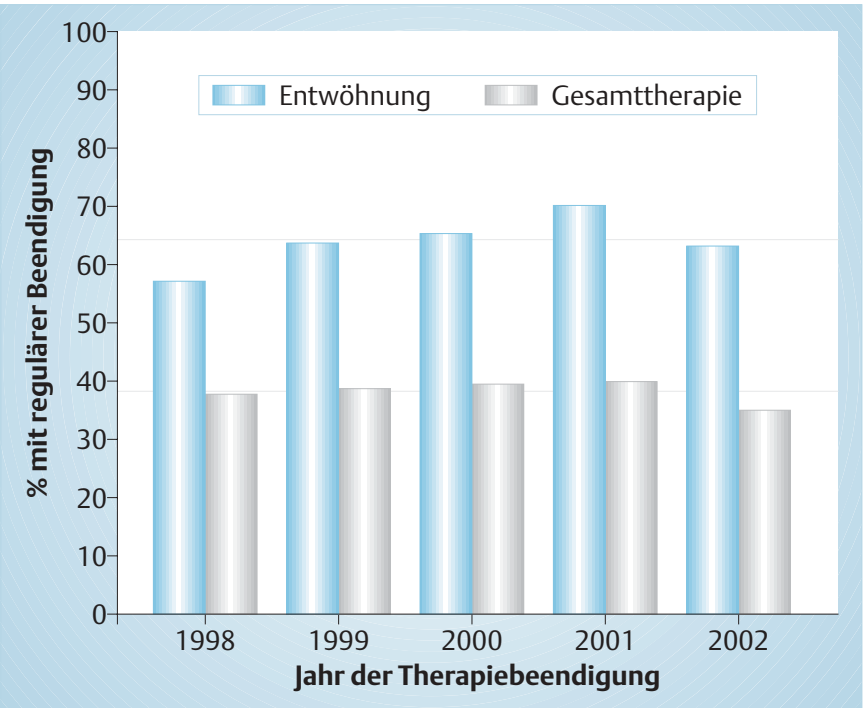

Abb. 1 Reguläre Beendigung der ersten Therapiestufe (Entwöhnung) bzw. der Gesamttherapie (Entwöhnung und interne Adaption) in Prozent; $\mathrm{n}=766$.

vorbereitet (Absicherung der Wohnsituation, der Finanzierung des Lebensunterhalts u. der Nachsorge) entlassen wurden.

39\% $(n=299)$ beendeten die Therapie auf eigenen Wunsch vorzeitig, und $23 \%(n=175)$ wurden von der Einrichtung entlassen.

Die mittlere Therapiezeit aller Klienten betrug 164 Tage $( \pm 121)$, der Medianwert lag bei 144 Tagen, die Therapiezeit der regulären Therapiebeender bei 284 Tagen ( \pm 78$) .43 \%$ waren länger als sechs Monate, $29 \%$ länger als neun Monate in der Therapie.

$27 \%$ aller vorzeitigen Therapiebeendigungen $(n=474)$ erfolgten bis zum Ende des ersten, 58\% bis zum Ende des dritten Monats.

\section{Integration in Arbeit und Ausbildung}

Nach fünfmonatiger Therapiezeit waren in der zweiten Therapiestufe $86 \%$ in Arbeit oder Ausbildung integriert (Mehrfachantworten): $52 \%$ waren erwerbstätig, $15 \%$ nahmen an berufsbildenden Maßnahmen (Berufsausbildung; Umschulung; Fachschule) teil, $10 \%$ besuchten allgemeinbildende Schulen, $24 \%$ absolvierten ein Praktikum oder einen Ausbildungsvorbereitungskurs. 14\% gelang keine beruflich-schulische Integration.

Im halben Jahr vor der Therapie waren 25\%, zum Zeitpunkt der Therapiebeendigung in der zweiten Therapiestufe $73 \%$ im Mittel seit 3,8 Monaten in Arbeit oder Ausbildung integriert ( $p<.001$; $\mathrm{n}=340$ ). Die Effektgröße betrug $d=1,11$ und ist als groß zu klassifizieren. 79\% der regulären und 53\% der vorzeitigen Therapiebeender in der zweiten Therapiestufe waren am Therapieende integriert $(\mathrm{p}<.001)$.

Im Detail (Abb. 2): $41 \%(\mathrm{n}=139)$ der Therapiebeender standen in einem Arbeitsverhältnis: $5 \%$ arbeiteten im erlernten Beruf und $22 \%$ als ungelernte Kraft auf dem ersten Arbeitsmarkt; $3 \%$ auf einer ABM-Stelle; $11 \%$ auf einer sozialversicherten „Hilfe-zur-Arbeit"-Stelle. $14 \%(n=46)$ nahmen an einer Berufsausbildung teil, $7 \%(n=25)$ gingen zur Schule, $11 \%(n=38)$ absolvierten ein Praktikum oder einen berufsbildenden Vorbereitungskurs über das 


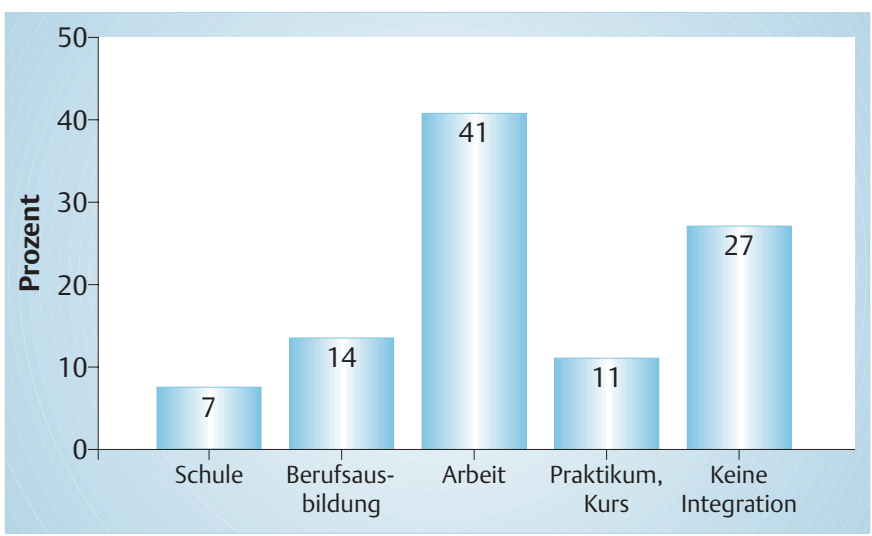

Abb. 2 Integration in Arbeit/Ausbildung bei Therapiebeendigung in der zweiten Therapiestufe in Prozent; $n=340$.

Arbeitsamt. 27\% ( $n=92)$ waren zum Auszugszeitpunkt nicht integriert, darunter ein Teil mit dem Beginn einer Maßnahme nach Therapiebeendigung.

In der gesamten Untersuchungsgruppe waren im halben Jahr vor der Therapie $26 \%$ zumindest kurzzeitig und bei Therapiebeendigung 50\% im Mittel seit 3,5 Monaten in Arbeit oder Ausbildung integriert (Effektgröße d=0,53; $\mathrm{n}=601$ mit Daten zu zwei Zeitpunkten).

\section{Weitere Ergebnisse}

Vor der Therapie lebten 39\% und bei Therapiebeendigung nach mindestens fünfmonatiger Therapiezeit $59 \%$ in eigenem Wohnraum $(n=339 ; p<.001)$, und zwar $80 \%$ der regulären und $42 \%$ der vorzeitigen Therapiebeender in der zweiten Therapiestufe $(\mathrm{p}<.001)$.

83\% der Therapiebeender in der zweiten Therapiestufe mit justiziellen Problemen verbesserten ihre juristische Situation. 92\% hatten sich um die Regulierung ihrer Schulden gekümmert. 93\% hatten während der Therapie (wieder) Kontakte zu drogenfreien Bekannten und Bezugspersonen aufgenommen.

\section{Veränderungen gemäß Klientenselbsteinschätzung}

Gemäß Klientenselbsteinschätzung konnten $71 \%$ sich durch die Therapie „stark“ bzw. „sehr stark“ in ihrer Persönlichkeit stabilisieren (Abb. 3). In freier Form beschrieben sie z. B. folgende Veränderungen $(n=259)$ :

„Habe gelernt, über Probleme mit anderen zu sprechen.“ „Ich kann Konflikte besser konstruktiv gestalten; ich kann mich besser beherrschen und raste nicht mehr so schnell aus; ich versuche mehr Verantwortung für mein Handeln zu übernehmen.“ „Kann wieder mit Menschen zusammen sein; kann mich wieder leiden; ich bin wieder wer; das Leben macht mir wieder Spaß.“ „Habe mich seelisch gut erholt; habe gelernt, in gewissen Situationen besser mit

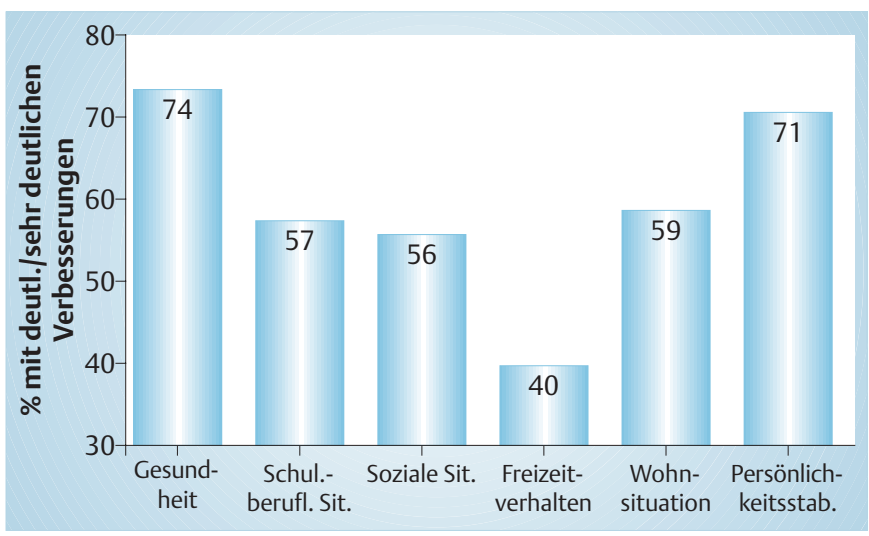

Abb. 3 Deutliche/sehr deutliche Verbesserungen in verschiedenen Bereichen gemäß Klienten-Selbsteinschätzung in Prozent; Therapiebeender der zweiten Stufe; $n=256-n=266$.

mir umzugehen; kann jetzt mal zur Ruhe kommen.“ „Ich gehe sehr bewusst durch das Leben, weiß genau, was ich mir auf dem Schirm behalten muss. Meine Stärken und Schwächen weiß ich, kann gelassen und flexibel auf Veränderungen reagieren.“ „Mehr Selbstbewusstsein ...“ „Habe teilweise gelernt, mich selbst mit schwierigen Themen auseinander zu setzen.“

57\% verbesserten gemäß Selbsteinschätzung ihre beruflichschulische Situation durch die Therapie „deutlich“ oder „sehr deutlich“ ( $n=265$ ). Sie schrieben z. B.:

„Habe über das Arbeitsamt einen Eignungstest gemacht und kann in näherer Zukunft eine Umschulung machen; seit ... arbeite ich über eine Zeitarbeit im Bürobereich.“ „ICh habe ein Gärtnerpraktikum gemacht und dadurch viel gelernt. Ich habe mich um einen Computerkurs beworben, kann dort aber erst im ... anfangen. "ICh habe nach langer Arbeitslosigkeit wieder einen Job; habe mir die Möglichkeit zur Umschulung als Elektroinstallateur erschlossen." „Umschulung zum Koch wieder aufgenommen.“ „Ich habe das erste Mal einen richtigen Job, der mir Spaß macht und wo ich mich auch fortbilden kann.“ „Arbeitsaufnahme in Wäscherei; eigenes Geld.“ „Festen Arbeitsplatz; Kontinuität; berufliche Integration; regelmäßiges Gehalt; Kontakte.“,Technikerschule ab September.“ „Habe eine Lehrstelle als Maler und Lackierer gefunden und kann in ... meine Prüfung ablegen; Zwischenprüfung ... theoretisch und praktisch bestanden!“ „Ich habe in der Therapie meinen Hauptschulabschluss nachgeholt; demnächst gehe ich zur Handelsschule."

Weiterhin: 74\% verbesserten durch die Therapie ihre körperlichgesundheitliche Verfassung, 59\% ihre Wohnsituation, 56\% ihre soziale Situation und $40 \%$ ihr Freizeitverhalten „deutlich“ oder „sehr deutlich“.

69-87\% der Klienten mit „deutlichen“ oder „sehr deutlichen Verbesserungen“ schätzten diese als „sehr wichtig“ ein.

Die regulären Therapiebeender erzielten gegenüber den vorzeitigen Therapiebeendern in der zweiten Therapiestufe größere „Allgemeine Therapieverbesserungen aus Klientensicht" ( $p<.001$; Effektgröße d =0,70). 


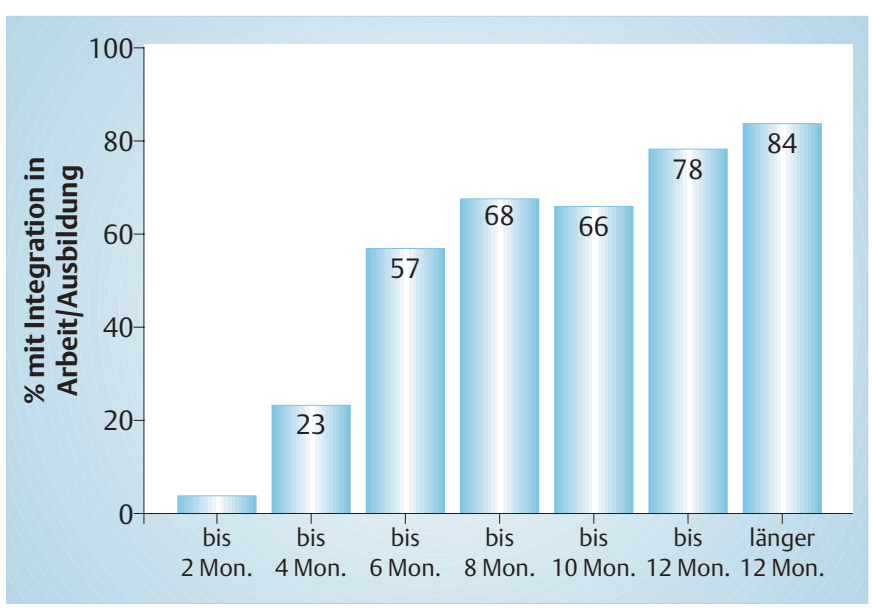

Abb. 4 Therapiezeit und Integration in Arbeit/Ausbildung; $n=625$.

Die Klientenselbsteinschätzungen stimmten mit den entsprechenden Mitarbeiterratings deutlich überein (Korrelation der Faktorwerte .76; $\mathrm{p}<.001$ ).

\section{Zufriedenheit mit den Therapieergebnissen}

$78 \%$ der Therapiebeender in der zweiten Stufe waren „zufrieden“ oder „sehr zufrieden“ mit den Ergebnissen ihrer Therapie. 19\% antworteten mit „weder noch“ und 3\% waren „unzufrieden“ oder „sehr unzufrieden“ $(n=264) .81 \%$ der regulären und $58 \%$ der vorzeitigen Therapiebeender waren „zufrieden“ oder „sehr zufrieden" mit den Ergebnissen ihrer Therapie $(p<.001)$.

\section{Therapieabschnitte mit stärksten Verbesserungen}

Hinsichtlich der Integration in Arbeit/Ausbildung am Therapieende erfolgte eine deutliche Verbesserung ab dem fünften Therapiemonat von $23 \%$ auf $57 \%$ Integrierter ( $<<.05 ;$ Abb. 4 ).

Größere „Allgemeine Therapieverbesserungen aus Mitarbeitersicht" wurden deutlich häufiger nach dem sechsten Therapiemonat (Anstieg von 33\% auf 64\%; $\mathrm{p}<.05$ ) erreicht, ebenso größere "Allgemeine Therapieverbesserungen aus Klientensicht“ (Anstieg von $27 \%$ auf $60 \%$; $<.05$ ). Nach dem 12 . Therapiemonat erzielten in den verschiedenen Ergebniskriterien noch 4\%-6\% Verbesserungen.

\section{Veränderungen in den Therapieergebnissen 1998-2002}

In den Therapieergebnissen hat es im Untersuchungszeitraum 1998-2002 keine signifikanten Veränderungen gegeben. Ausnahmen ( $\mathrm{p}<.05$ ): Der Anteil mit schulischer Integration bei Beendigung der zweiten Therapiestufe war von 20\% (1998) auf 2\% (2002) zurückgegangen, der Anteil mit Kursen/Vorbereitungskursen ist von 3\% (1998) auf 18\% (2002) gestiegen. Die regulären Therapiebeender zogen in den letzten drei Jahren gegenüber 1998/1999 seltener am Therapieende in eigenen Wohnraum.

\section{Prädiktion von Therapieergebnissen}

\section{Therapieergebnisse und Klientenmerkmale}

Es wurden jeweils zunächst Einzelzusammenhänge zwischen 65 Klientenmerkmalen und der Art der Therapiebeendigung (regulär vs. vorzeitig) in einer Reha-Einrichtung überprüft und dann in der anderen Einrichtung kreuzvalidiert. Folgende Merkmale korrelierten signifikant in beiden Therapieeinrichtungen schwach (Cramer-V .10-.22) mit der Art der Therapiebeendigung: Klienten beendeten häufiger die Therapie regulär, wenn sie eine abgeschlossene Berufsausbildung hatten, die Therapie nicht auf Basis des $§ 35$ BtmG (Aussetzung der Strafvollstreckung) begannen, bislang noch nicht inhaftiert waren, die Therapie ohne starken Justizdruck begannen, keine Haftzeiten von mehr als fünf Jahren hatten bzw. nicht direkt aus der Haft aufgenommen worden waren. Diese Variablen wurden in eine logistische Regression [26] einbezogen mit der Art der Therapiebeendigung als Kriterium. Mit der logistischen Regression soll überprüft werden, wie bedeutsam die einzelnen Variablen für die Vorhersage der Art der Therapiebeendigung sind. Ergebnis: Es gelang nur bei $64 \%$ der Fälle, die Art der Therapiebeendigung richtig vorherzusagen. Die Verbesserung gegenüber der Ausgangsklassifikation betrug 1,6\%. Die Art der Therapiebeendigung konnte durch die einbezogenen Klientenmerkmale nur geringfügig besser als per Zufallsprognose vorhergesagt werden.

Auch andere Ergebniskriterien, so Frühabbrüche innerhalb der ersten 90 Tage, die Art der vorzeitigen Therapiebeendigung (Abbruch vs. Entlassung), die Integration in Arbeit oder Ausbildung am Therapieende, „Allgemeine Therapieverbesserungen aus Mitarbeitersicht“ oder „Allgemeine Therapieverbesserungen aus Klientensicht" standen in keinem oder nur schwachem (max. .20) Zusammenhang mit Klientenmerkmalen zu Therapiebeginn.

\section{Therapieergebnisse und Prozessmerkmale}

Als Therapieprozessmerkmale wurden die in der Eingangshypothese angeführten Merkmale „Häufigkeit der Arbeit mit Rückfall“, „Integration in Arbeit/Ausbildung am Anfang der zweiten Therapiestufe" und die Therapiezeit berücksichtigt. Zusätzlich wurden die in der Patientennachbefragung des VDR [58] berücksichtigten „Therapieprozessmerkmale aus Klientensicht“ und als einrichtungsspezifisches Merkmal die „Intensität der schulischberuflichen Vorbereitung in der ersten Therapiestufe“ gemäß Mitarbeitereinschätzung einbezogen.

Bei getrennten Analysen in beiden Reha-Einrichtungen gab es in beiden Einrichtungen jeweils folgende Zusammenhänge (alle $\mathrm{p}<.001$; Koeffizient jeweils Cramer-V (zusammengefasste Daten beider Einrichtungen):

Arbeit mit Rückfall: Mit Klienten, die größere „Allgemeine Therapieverbesserungen aus Mitarbeitersicht“ erzielt hatten, war häufiger mit Rückfall gearbeitet worden (.20). Ansonsten stand die Häufigkeit der Arbeit mit Rückfall in keinem Zusammenhang mit den Therapieergebnissen.

Intensität der schulisch-beruflichen Vorbereitung in der ersten Therapiestufe: Die regulären Therapiebeender hatten sich bereits in der ersten Therapiestufe gemäß Mitarbeitereinschätzung intensiver mit ihrer beruflichen oder schulischen Perspektive auseinander gesetzt (.48), ebenso die am Therapieende in Arbeit/Ausbildung Integrierten (.59) sowie Therapiebeender mit größeren „Allgemeinen Therapieverbesserungen aus Mitarbeitersicht" (.58) oder mit größeren „Allgemeinen Therapieverbesserungen aus Klientensicht" (.42). 
Integration in Arbeit/Ausbildung am Anfang der zweiten Therapiestufe: Die regulären Therapiebeender (.41), die zum Auszugszeitpunkt beruflich oder schulisch Integrierten (.72) sowie Therapiebeender mit größeren „Allgemeinen Therapieverbesserungen aus Mitarbeitersicht“(.39) waren bereits am Anfang der zweiten Therapiestufe häufiger in Arbeit oder Ausbildung integriert.

Therapieprozessmerkmale aus Klientensicht: In einer Teilstichprobe $(n=170)$ hatten die regulären Therapiebeender den Therapieprozess positiver wahrgenommen (.36), ebenso Therapiebeender mit größeren „Allgemeinen Therapieverbesserungen aus Klientensicht“(.35).

Therapiezeit: Klienten mit längerer Therapiezeit hatten am Therapieende eine bessere Integration in Arbeit oder Ausbildung erreicht (partielle Korrelation unter Ausschaltung der Art der Therapiebeendigung; .28), sie hatten größere „Allgemeine Therapieverbesserungen aus Mitarbeitersicht“ (.47) und ebenfalls aus Klientensicht (.33) erzielt.

Multivariate Analysen: Über logistische Regression mit den in den bivariaten Analysen in beiden Reha-Einrichtungen in signifikantem Zusammenhang stehenden Prozessmerkmalen als Prädiktoren konnte die Art der Therapiebeendigung (regulär vs. vorzeitig) in $78 \%$ der Fälle bzw. die Integration in Arbeit/Ausbildung am Therapieende bei $89 \%$ richtig vorhergesagt werden. Die Verbesserung der Vorhersagegenauigkeit um 18\% bzw. 35\% ging nahezu ausschließlich auf die „Integration in Arbeit/Ausbildung am Anfang der zweiten Therapiestufe“ zurück.

Die „Allgemeinen Therapieverbesserungen aus Mitarbeitersicht“ (größere vs. geringere) konnten in 83\% der Fälle und die „Allgemeinen Therapieverbesserungen aus Klientensicht“ ( $n=143)$ in $76 \%$ richtig klassifiziert werden. Die Erhöhung der Vorhersagegenauigkeit um 29\% bzw. 22\% gelang nahezu allein durch die Therapiezeit als Prädiktor.

\section{Diskussion}

Im Vergleich mit den sich an der bundesweiten SEDOS- bzw. EBISDokumentation beteiligenden Einrichtungen im entsprechenden Zeitraum [27 - 31], aber auch im Vergleich zu den in der Hamburger Basisdatendokumentation [32-36] beschriebenen Klienten, die sich im ambulanten Beratungs- und Betreuungssystem befanden, hatten die Klienten der Hamburger Sozialtherapeutischen Wohngemeinschaften die Therapie insgesamt mit deutlich stärkerer psychosozialer Problembelastung begonnen, z.B. mit besonders langer Drogenabhängigkeit, mehr Behandlungserfahrung, überdurchschnittlichen Defiziten im Bildungs- und Ausbildungsbereich, höherer justizieller Belastung oder sehr prekärer sozialer Situation.

Das hohe Ausmaß der erlebten körperlichen und sexuellen Gewalterfahrungen entsprach etwa dem in der Hamburger Basisdokumentation berichteten [36] bzw. dem einer neueren Studie von Frauen in Suchtfachkliniken [37].

Bemerkenswert ist, dass es im 5-Jahres-Zeitraum in den untersuchten Klientenmerkmalen nahezu keine Veränderungen beim aufgenommenen Personenkreis gegeben hat. In einer umfangreichen Schweizer Studie über mehr als 3600 abstinenzorientierte Therapien wurde im Untersuchungszeitraum 1995-1999 ebenfalls keine praktisch relevante Veränderung der psychosozialen Eingangsvoraussetzungen festgestellt [38].

Vergleicht man die Klienten des Untersuchungszeitraums allerdings mit denen aus länger zurückliegenden Jahren [7, 9], so waren sie deutlich älter, etwa doppelt so lange drogenabhängig und mit häufigeren und längeren Hafterfahrungen belastet. Sie hatten zugleich häufigere Therapievorerfahrungen, vor allem waren sie deutlich häufiger substituiert gewesen. Ihre gesundheitlichen Beeinträchtigungen waren größer.

Etwa zwei Drittel beendeten die fünfmonatige Entwöhnungstherapiephase und vier von zehn die Gesamttherapie mit interner Adaption regulär. Die regulären Therapiebeender waren knapp zehn Monate, alle Klienten etwa fünfeinhalb Monate in der Therapie. Die Haltequote, definiert durch die Anteile regulärer Beendigung und die Therapiezeit, lag damit insgesamt eher im überdurchschnittlichen Bereich, wobei es unter Berücksichtigung des überdurchschnittlich belasteten Personenkreises und der besonderen konzeptionellen Ausrichtung auf Integration in Arbeit oder Ausbildung nur äußerst eingeschränkte Vergleichsmöglichkeiten gibt [27-31, 39-50].

Trotz der erheblichen Defizite in der schulischen und beruflichen Qualifikation konnten vergleichsweise [27-31, 39-41] sehr gute Ergebnisse bei der konzeptionell angestrebten Integration in $\mathrm{Ar}$ beit oder Ausbildung erreicht werden: In der zweiten Therapiestufe nach mindestens fünf Monaten waren $86 \%$, bei Beendigung der Therapie in der zweiten Therapiestufe 73\% im Mittel seit knapp vier Monaten in Arbeit oder Ausbildung integriert. Die wesentlichen Therapieergebnisse waren im Studienzeitraum weitgehend konstant.

Nach den früheren Veröffentlichungen [7, 9, 16, 17] wurde in der aktuellen Untersuchung unter Einbeziehung einer sehr großen Zahl von Therapieverläufen erneut nachgewiesen, dass die Integration von Drogenabhängigen auch mit sehr ungünstigen Eingangsvoraussetzungen in normale Ausbildungs- und Arbeitsverhältnisse prinzipiell möglich ist, wenn konzeptionell dieser Zielbereich entsprechend rechtzeitig und systematisch berücksichtigt wird.

Es wurde zugleich gezeigt, dass es möglich ist, die schulisch-berufliche Integration während der stationären Therapie im wechselseitigen Kontext mit der Klärung anderer wichtiger drogentherapeutischer Fragen zu erreichen. Dieses Ergebnis wurde erreicht trotz deutlich verschlechterter gesellschaftlicher Rahmenbedingungen insbesondere im Arbeits- und Beschäftigungsbereich.

Bei der Integration in Arbeit oder Ausbildung erfolgten in den letzten Jahren qualitative Verschlechterungen: Sehr viel weniger besuchten eine Schule, deutlich mehr nahmen an Vorbereitungskursen teil. Durch den in den letzten Jahren in Hamburg erfolgten Abbau von Tagesschulangeboten für Volljährige ist der Anteil mit schulischer Integration ganz erheblich zurückgegangen, obwohl gut ein Drittel der Klienten keinen Hauptschulabschluss 
aufwies. Gleichermaßen sind auf dem normalen Ausbildungsmarkt wie auch bei alternativen Beschäftigungsträgern die Berufsausbildungsangebote für Jüngere erheblich reduziert worden und in jüngster Zeit werden Umschulungen nahezu nicht mehr bewilligt [51]. Während die Rentenversicherungsträger in wachsendem Maße Wert auf die reale berufliche Eingliederung legen $[14,15,44,52]$ und sogar überlegt wird, Erfolge in diesem Bereich besonders zu honorieren [53], werden durch die gesellschaftlichen Rahmenbedingungen die für Rehabilitanden zu überwindenden Mauern deutlich höher.

Zum Erreichen deutlicher Therapieverbesserungen bedarf es offensichtlich bestimmter Mindesttherapiezeiten, und diese sind je nach Ergebniskriterium unterschiedlich und ebenfalls abhängig von der Einrichtungskonzeption, die vorgibt, wann bestimmte Therapieschritte erfolgen können. In den Sozialtherapeutischen Wohngemeinschaften wurden nach einer Therapiezeit von etwa fünf bis sieben Monaten die deutlichsten Ergebnisverbesserungen in verschiedenen Bereichen erzielt. Dieses Ergebnis kommt dem Resümee von Roch et al. [18] bzw. Sonntag und Künzel [54] recht nahe, die unter Auswertung inzwischen länger zurückliegender Studien von einem ähnlichen Zeitraum ausgingen. Nach dieser Mindesttherapiezeit verbesserten sich die Therapieergebnisse kontinuierlich weiter. Erst nach einem Jahr gab es kaum noch Verbesserungen. Es scheint die flexible Budgetregelung der Rentenversicherungsträger mit einer Regelzeit einschließlich Adaption von 10 Monaten durch die vorliegenden Ergebnisse untermauert zu werden. Eine weitere Verkürzung der Therapiezeit wäre kontraproduktiv für Ausstiegs- und Integrationsbemühungen bei einem Personenkreis, der ganz erheblich sozial und biografisch belastet ist. Am Rande: $87 \%$ der Rehakosten entfielen auf die Therapiebeender in der zweiten Therapiestufe mit interner Adaption, also auf jenen Personenkreis, der diese für deutliche Verbesserungen notwendige Mindesttherapiezeit erreicht hatte.

Es konnten keine relevanten Beziehungen zwischen Anamnesedaten der Klienten und den verschiedenen Therapieergebniskriterien nachgewiesen werden. Die Prognose des individuellen Therapieverlaufs anhand von Anamnesemerkmalen oder Behandlungsvorerfahrungen (z. B. der Anzahl oder Dauer von Therapieaufenthalten) ist so gut wie nicht möglich. Insgesamt wird der in früheren Studien gefundene Forschungsstand gestützt, dass zur Vorhersage von Therapieergebnissen der Beitrag von Klientenmerkmalen gering ist $[4,5,9,11,17]$. Es bleibt offen, ob durch Einbeziehung anderer diagnostischer Daten, z. B. zur psychischen oder somatischen Komorbidität, eine bessere Vorhersage möglich ist.

Die regulären Therapiebeender hatten erwartungsgemäß hinsichtlich „harter“ Ergebnisvariablen sowie in Mitarbeiter- und Klientenselbsteinschätzungen bessere Therapieergebnisse erzielt als vorzeitige. Dabei darf einerseits ein gewisser Einfluss auf die Ratingergebnisse durch die Kenntnis der Art der Therapiebeendigung vermutet werden, andererseits gibt es eine deutliche Konsistenz zwischen den methodisch unterschiedlich erhobenen Ergebnissen.
Um Therapieergebnisse, insbesondere die Haltequote, weiter zu verbessern, scheint es fruchtbarer, sich den Therapieprozessen stärker zuzuwenden: Dabei ging die häufigere Auseinandersetzung mit Abstinenzverletzungen entgegen der Annahme nicht einher mit schlechteren Therapieergebnissen; es zeigte sich vielmehr ein schwacher Zusammenhang, dass bei Therapiebeendern mit besseren Therapieergebnissen häufiger mit Rückfall gearbeitet worden war. Zu berücksichtigen ist dabei, dass nach therapeutischen Verlaufskriterien eine Auswahl bezüglich der Fortsetzung der Therapie getroffen wurde, so dass wahrscheinlich eher mit stärker motivierten Klienten weiter gearbeitet wurde. Das vorliegende Ergebnis entspricht dem zweier umfangreicher Schweizer Studien [55, 56]. Hingegen hatten in den SEDOS-Dokumentationen 1998 und 1999 die vorzeitigen Therapiebeender mehr als doppelt so viele Rückfälle wie die regulären [27, 28].

Die Therapiezeit, ferner die Integration in Arbeit/Ausbildung etwa in der Therapiemitte standen, wie eingangs angenommen, am deutlichsten in Zusammenhang mit besseren Therapieergebnissen.

Die Integration in Arbeit oder Ausbildung gelingt in der Regel nur dann, wenn sie entsprechend den individuellen Voraussetzungen der Klienten zielgerichtet gut vorbereitet und begleitet wird. Als sehr wichtig hat sich dabei die personelle und konzeptionelle Kontinuität zwischen der Entwöhnungsphase und der internen Adaption erwiesen. Zur fachlichen Begleitung und personenbezogenen Beratung ist es unabdingbar, dass die für die Berufsorientierung zuständigen sozialpädagogischen Mitarbeiter sich fortbilden hinsichtlich der gesetzlichen Veränderungen im Arbeits-, Ausbildungs- und Weiterbildungsbereich bzw. Netzwerke mit diesem spezifischen Hintergrund nutzen können. Wichtig ist, dass sie eine genaue Kenntnis der im Arbeits- und Ausbildungsbereich engagierten Betriebe, Projekte und Einrichtungen in der Region haben. Nur so kann zielgerichtet und fundiert beraten und aktiv unterstützt werden. Für die Klienten finden wesentliche Entscheidungen statt: Den Weg in Richtung Arbeit oder Ausbildung zu gehen, impliziert für viele, sich mehr von der Szenelebensweise abzuwenden. Dabei werden sie unterstützt und in Krisensituationen aufgefangen durch die Therapeutische Gemeinschaft.

Der Zusammenhang zwischen Therapiezeit und Therapieergebnissen dürfte so zu verstehen sein, dass es auf die Erfahrungen und Fortschritte in dieser Zeit ankommt [57]. In neueren angloamerikanischen Studien wurde auf die Bedeutung der Behandlungsintensität, der Therapiebeteiligung, des Klienten-Engagements, der Therapeut-Klient-Beziehung, der Therapiezufriedenheit als wichtige Prozessvariablen für Haltequote und Therapieergebnisse hingewiesen. Wahrscheinlich ist die Therapiezeit auch mit komplexeren Ausleseprozessen verbunden: So ist anzunehmen, dass motivierte Klienten sich mehr engagieren, dadurch mehr Fortschritte machen und so länger in der Therapie verbleiben. Wiederum haben therapeutische Interventionen Einfluss auf den Motivationsprozess und dieser wiederum auf die Aufenthaltsdauer [54]. 
Literatur

${ }^{1}$ Ludwig G, Vormann G. Katamnestische Untersuchung für die therapeutischen Gemeinschaften der STEP - Gem. Gesellschaft für Sozialtherapie u. Pädagogik mbH für den Zeitraum 1973-1980. Informationen aus der Therapiekette Niedersachsen. Hannover, 1981: 6, 1: $29-39$

2 Raschke P, Schliehe F. Vorstudie über den Erfolg von Therapie und Rehabilitation von Drogenabhängigen am Beispiel des „Hammer Modells“. Bielefeld: IDIS, 1982

${ }^{3}$ Therapiehilfe e.V. (Hrsg). Do-it-Journal. Hamburg, 1987

${ }^{4}$ Vollmer HC, Ellgring H. Die Vorhersage der vorzeitigen Therapiebeendigung bei Drogenabhängigen. Suchtgefahren 1988; 34 (4): 273-284

${ }^{5}$ Herbst K. Verlaufsanalyse bei Drogenabhängigen nach stationärer Behandlung. Sucht 1992; 38: 147-154

${ }^{6}$ Groenemeyer A, Birtsch V. Frauen und Männer mit Kindern in der Drogentherapie. Erfahrungen und Evaluation der Drogenhilfe Tübingen Haus Friedrichshof. Frankfurt, 1991

7 Thiel G. Erfahrungen bei der schulischen und beruflichen Integration langjährig Drogenabhängiger. Sucht 1995; 41 (5): 334-341

8 Drogenhilfe Bremen (Hrsg). Festschrift 25 Jahre Drogenhilfe Bremen. www.hohehorst.de/information/Festschrift

9 Thiel G, Pölckow M. Therapieverlauf, schulisch-berufliche Integration, Katamnese und Prozesszusammenhänge bei 100 drogenabhängigen Personen in einer Sozialtherapeutischen Wohngemeinschaft. Wiener Zeitschrift für Suchtforschung 1998; 21 (1): $37-48$

10 Simon R, Palazetti M. Jahresstatistik 1997 der stationären Suchtkrankenhilfe in der Bundesrepublik Deutschland. SEDOS-Berichte. Hamm, 1998: Bd. 5

${ }^{11}$ Küfner H, Denis A, Roch I et al. Stationäre Krisenintervention be Drogenabhängigen. Ergebnisse der wissenschaftlichen Begleitung des Modellprogramms. In: Bundesministerium f. Gesundheit (Hrsg). Baden-Baden, 1994

${ }^{12}$ Sickinger R. Ausstieg aus der Heroinabhängigkeit. Freiburg1994

${ }^{13}$ Henkel D. Zur epidemiologischen, ätiologischen und rehabilitativen Relevanz der Arbeitslosigkeit für den Alkoholismus: Empirische Fakten, theoretische Reflexionen und politische Forderungen. In: Fachverband Sucht e.V. (Hrsg). Sucht und Erwerbsfähigkeit. Wie erfüllen Rehabilitationseinrichtungen ihren Auftrag? Beiträge des 8. Heidelberger Kongresses 1995. Geesthacht, 1996: 307-329

${ }^{14}$ Fachverband Sucht e.V. (Hrsg). Sucht und Erwerbsfähigkeit. Wie erfüllen Rehabilitationseinrichtungen ihren Auftrag? Beiträge des 8 . Heidelberger Kongresses 1995. Geesthacht, 1996

${ }^{15}$ Schallenberg H. Wiederherstellung der Erwerbsfähigkeit im Rahmen medizinischer Rehabilitation. In: Fachverband Sucht e.V. (Hrsg). Sucht und Erwerbsfähigkeit. Wie erfüllen Rehabilitationseinrichtungen ihren Auftrag? 8. Heidelberger Kongreß 1995. Geesthacht, 1996: $133-140$

16 Thiel G. Allgemeine und differentielle Katamneseergebnisse in einer Hamburger Sozialtherapeutischen Wohngemeinschaft für Drogenabhängige. Wiener Zeitschrift für Suchtforschung 1995; 18 (3): 17 - 25

17 Thiel G, Ackermann R. Therapievoraussetzungen und Ergebnisse. Evaluation der stationären Drogentherapie bei Jugend hilft Jugend e. V. im Jahre 1996. Abschlußbericht. Hamburger Fortbildungsinstitut Drogen und Aids. Hamburg, 1998

${ }^{18}$ Roch I, Küfner H, Arzt J et al. Empirische Ergebnisse zum Therapieabbruch bei Drogenabhängigen: Ein Literaturüberblick. Sucht 1992; 38: $304-322$

${ }^{19}$ Bleiberg JL, Devlin P, Croan J et al. Relationship between treatment length and outcome in a Therapeutic Community. International Journal of the Addictions 1994; 39 (6): 729 - 740

${ }^{20}$ Condelli WS, Hubbard RL. Relationship Between Time Spent in Treatment and Client Outcomes From Therapeutic Communities. Journal of Substance Abuse Treatment 1994; 11 (1): 25-33

${ }^{21}$ De Leon G. Therapeutic Communities. In: Galanter M, Kleber HD (eds). The American psychiatric press textbook of substance abuse treatment. Washington: American Psychiatric Press, 1994: 391 - 414

${ }^{22}$ McCusker J, Bigelow C, Frost R et al. The Effects of Planned Duration of Residential Drug Abuse Treatment on Recovery and HIV Risk Behavior. American Journal of Public Health 1997; 87 (10): 1637-1644

${ }^{23}$ Projekt Jork - Jugendhilfe e.V. (Hrsg). Wissenschaftliche Konzeption. Hamburg, 2003

24 Jugend hilft Jugend e.V. (Hrsg). Konzept zur stationären Therapie mit Drogenabhängigen im Träger Jugend hilft Jugend e.V. Hamburg, 1999
${ }^{25}$ Bortz J, Döring N. Forschungsmethoden und Evaluation. Berlin/Heidelberg, 1995

${ }^{26}$ Bühl A, Zöfel P. SPSS für Windows. Version 6.1. Praxisorientierte Einführung in die moderne Datenanalyse. Bonn, 1995

27 Simon R, Palazetti M. Jahresstatistik 1998 der stationären Suchtkrankenhilfe in der Bundesrepublik Deutschland. Sucht 1999; 44 (Sonderheft 1): 60-94

${ }^{28}$ Strobl M, Lange SN, Zahn H. SEDOS ‘99. Stationäre Suchtkrankenhilfe. Einrichtungstyp-Auswertung FKH Illegale Drogen. In: SEDOS-AG bei der Deutschen Hauptstelle gegen die Suchtgefahren (Hrsg). München: IFT, 2000

${ }^{29}$ Strobl M, Pelzel KH, Bader G et al. EBIS-S 2000. Jahresauswertung stationäre Suchtkrankenhilfe. In: EBIS-AG bei der Deutschen Hauptstelle gegen die Suchtgefahren (Hrsg). München: IFT, 2001

30 Strobl M, Klapper J, Pelzel KH et al. Suchthilfestatistik für Deutschland. Tabellenband für die stationäre Suchtkrankenhilfe. München: IFT, 2002

31 Strobl M, Klapper J, Pelzel KH et al. Suchthilfestatistik 2002 für Deutschland. Tabellenband für stationäre Einrichtungen, alle Bundesländer. München: IFT, 2003

${ }^{32}$ Schmid M, Simmedinger R, Vogt I. Ambulante Suchthilfe in Hamburg. Statusbericht 1998 zur Hamburger Basisdatendokumentation im ambulanten Suchthilfesystem. Frankfurt am Main: ISS-Eigenverlag, 1999

${ }^{3}$ Schmid M, Simmedinger R, Vogt I. Ambulante Suchthilfe in Hamburg. Statusbericht 1999 zur Hamburger Basisdatendokumentation im ambulanten Suchthilfesystem. Frankfurt am Main: ISS-Eigenverlag, 2000

${ }^{34}$ Simmedinger R, Schmid M, Vogt I. Ambulante Suchthilfe in Hamburg. Statusbericht 2000 zur Hamburger Basisdatendokumentation im ambulanten Suchthilfesystem. Frankfurt am Main, 2001

${ }^{35}$ Martens MS, Verthein U, Degkwitz P. 2001. Ambulante Suchthilfe in Hamburg. Statusbericht der Hamburger Basisdatendokumentation. In: BADO e. V. (Hrsg). Hamburg, 2003

36 Martens MS, Lorenzen J, Verthein U et al. 2002. Ambulante Suchthilfe in Hamburg. Statusbericht der Hamburger Basisdatendokumentation. In: BADO e. V. (Hrsg). Hamburg, 2003

37 Zenker C, Bammann K, Jahn I. Ursachen und Differenzierungen der Abhängigkeitserkrankungen bei Frauen. Sucht aktuell 2003; 10 (2): $15-20$

38 Grichting E, Uchtenhagen A, Reichlin M. Stellenwert und Klientele stationärer abstinenzorientierter Therapien für Drogenabhängige in der Schweiz. Abschlußbericht der Koordinierungsstelle des Forschungsverbundes stationäre Suchttherapie FOS zur „FOS-Basisdokumentation“" in den Jahren 1997-2000. ISF-Bericht 131. Zürich, 2002. http://www.suchtforschung.ch/

${ }^{39}$ Küfner H, Vogt M, Weiler D. Medizinische Rehabilitation und Methadon-Substitution. Hohengehren, 1999

40 STEP - Gesellschaft für Sozialtherapie und Pädagogik mbH (Hrsg). Jahresbericht 2000. Hannover, 2000

41 STEP - Gesellschaft für Sozialtherapie und Pädagogik mbH (Hrsg). 30 Jahre STEP. Jahresbericht 2001. Hannover, 2001

${ }^{42}$ Schröder W. Integrierte Behandlung von Alkohol-, Medikamentenund Drogenabhängigen. In: Fachverband Sucht e.V. (Hrsg). Die Zukunft der Suchtbehandlung. Trends und Prognosen. Geesthacht, 2002: 93-99

${ }^{43}$ Löhrer F. Psychotische und nichtpsychotische Substanzgebraucher: Eine prospektive Studie zum Reha-Verlauf und -Ergebnis. In: Fachverband Sucht e. V. (Hrsg). Die Zukunft der Suchtbehandlung. Trends und Prognosen. Geesthacht, 2002: 267-273

${ }^{44}$ Schillinger H. Die Zukunft der Rehabilitation und der Suchtbehandlung: Trends und Prognosen aus Sicht der Rentenversicherung. In: Fachverband Sucht e.V. (Hrsg). Die Zukunft der Suchtbehandlung. Trends und Prognosen. Geesthacht, 2002: 75-92

${ }^{45}$ Senatsverwaltung für Schule, Jugend und Sport, Referat Drogen und Sucht (Hrsg). Jahresbericht 2000 zur Drogen- und Suchtproblematik in Berlin. Berlin, 2001. http://www.berlin.de/imperia/md/content/ sengsv/drogen_und_sucht

${ }^{46}$ Kunz D. Medizinische Rehabilitation/Entwöhnungsbehandlung. In: Jugendberatung und Jugendhilfe e.V. (Hrsg). Jahresbericht 2000 des Suchthilfeverbundes Jugendberatung und Jugendhilfe e.V. Frankfurt 2001: $37-39$

47 Siegele R. Qualitätssicherung in der Drogenarbeit: Eine katamnestische Untersuchung in der therapeutischen Gemeinschaft Drogenhilfe Tübingen - Friedrichshof. Diplomarbeit. Studiengang Sozialwesen. Fachhochschule Würzburg - Schweinfurt, 2001 
${ }^{48}$ Kurz V. Katamneseuntersuchung des Jahrgangs 1999 am Zentrum III der Drogenhilfe Tübingen e.V. - Friedrichshof. Diplomarbeit. Psychologisches Institut der Eberhard Karls Universität Tübingen. Tübingen, 2002

${ }^{49}$ Reininger S. Qualitätssicherung in der Drogenhilfe. Katamneseuntersuchung des Jahres 2000 der therapeutischen Gemeinschaft Drogenhilfe Tübingen e.V. - Friedrichshof. Diplomarbeit. Institut für Psychologie der Humboldt-Universität zu Berlin. Berlin, 2003

${ }^{50}$ Müller-Fahrnow W, Ilchmann U, Kühner S et al. Sucht-Rehabilitation in Deutschland: Bestandsaufnahme und Perspektiven. Eine Expertise des Lehrstuhls für Versorgungssystemforschung - Humboldt-Universität zu Berlin. In: Verband Deutscher Rentenversicherungsträger (Hrsg). Frankfurt am Main, 2002

${ }^{51}$ Fachverband Drogen und Rauschmittel (Hrsg). Problemanzeige: Berufliche Eingliederung von Suchtkranken. FDR Berichte 2003; 57: 3-4

52 Wolfsburger Resolution zur Förderung der Teilhabe am Arbeitsleben von suchtkranken Menschen vom 4.12.2003. www.bfa.de

${ }^{53}$ Mootz U. Gesamtrehabilitationsstrategie aus Sicht der BfA und deren Bedeutung für die Behandlung Abhängigkeitskranker. In: Fachverband Sucht e.V. (Hrsg). Rehabilitation Suchtkranker - mehr als Psychotherapie. Schriftenreihe des Fachverbandes Sucht e.V., 24. Geesthacht, 2001: 75-86

${ }^{54}$ Sonntag D, Künzel J. Hat die Therapiedauer bei alkohol- und drogenabhängigen Patienten einen positiven Einfluss auf den Therapieerfolg? Sucht 2000; 46 (Sonderheft 2): 92 - 176
${ }^{55}$ Schaaf S, Reichlin M, Grichting E et al. Der Forschungsverbund stationäre Suchttherapie FOS im Jahr 2000. Tätigkeitsbericht und Jahresstatistik. Vergleich von Klienten und Klienten mit unterschiedlichem Konsummuster. In: Koordinierungsstelle des FOS (KOFOS) (Hrsg). Forschungsbericht aus dem Institut für Suchtforschung Nr. 119. Zürich, 2001. (www.suchtforschung.ch)

${ }^{56}$ Schaaf S, Reichlin M, Hampson D et al. Der Forschungsverbund stationäre Suchttherapie FOS im Jahr 2001. Tätigkeitsbericht und Jahresstatistik. Vergleich von Klienten mit unterschiedlicher Behandlungserfahrung. Koordinierungsstelle des FOS (KOFOS) (Hrsg). Forschungsbericht aus dem Institut für Suchtforschung Nr. 146. Zürich, 2002 (www.suchtforschung.ch)

57 Toumbourou JW, Hamilton M, Fallon B. Treatment level progress and time spent in treatment in the prediction of outcomes following drugfree therapeutic community treatment. Addiction 1998; 93 (7): $1051-1064$

${ }^{58}$ Verband Deutscher Rentenversicherungsträger (Hrsg). Das Qualitätssicherungsprogramm der gesetzlichen Rentenversicherung in der medizinischen Rehabilitation. Instrumente und Verfahren. Januar 2000. DRV-Schriften. Frankfurt am Main, 2000: Band 18 